\title{
Experimental Infection of Adult Axenic Rats With Parker's Rat Coronavirus
}

\author{
By \\ P. N. BHaTt and R. O. JACOBY \\ Section of Comparative Medicine, Yale University School of Medicine, \\ New Haven, Connecticut, U.S.A. \\ With 5 Figures
}

Accepted March 31, 1977

\begin{abstract}
Summary
The pathogenesis of Parker's Rat Coronavirus (PRCV) was studied in axenic CD rats. Three to four 9 to 10 week old rats were euthanized daily for eight days after intranasal inoculation. Rats remained free of clinical disease. Virus was recovered from the nasopharynx and trachea after twenty-four hours and from the lung by day three but was not detected in respiratory tract after seven days. Viral antigen was detected by indirect immunofluorescence in the mucosal epithelium of upper respiratory tract and in pulmonary alveolar septae from day two to six postinoculation. Acute rhinitis developed by day two and was associated with mild focal necrosis of respiratory mucosal epithelium. Mild nonsuppurative tracheitis and multifocal interstitial pneumonia appeared by day five and persisted through day eight. Dacryoadenitis did not occur, sialoadenitis was detected in only three rats and virus was recovered from only one submaxillary salivary gland. This experiment indicates that PRCV can be a primary pathogen for the respiratory system of adult rats. In contrast to sialodacryoadenitis (SDA) virus the tropism of PRCV for salivary and lacrimal glands is low.
\end{abstract}

\section{Introduction}

Coronavirus infection is common in laboratory rats and two antigenically related viruses, sialodacryoadenitis virus (SDAV) and Parker's rat coronavirus (PRCV), have been isolated from naturally-infected rats $(2,7)$. SDAV causes severe self-limiting sialodacryoadenitis in naturally-infected or experimentallyinoculated adult rats $(4,5)$ and recent evidence indicates that SDAV is associated with naturally-occurring keratoconjunctivitis in rats (6). PRCV was originally isolated from the lungs of rats with a high incidence of complement-fixing (CF) 
antibody to mouse hepatitis virus (MHV) (7). In contrast to SDAV, natural infection with PRCV is reportedly asymptomatic in adults, but experimentallyinoculated neonates developed lethal interstitial pneumonia (7). This report described experimental PRCV infection of adult CD rats. Results indicate that PRCV is pathogenic for the respiratory system.

\section{Materials and Methods}

Fifteen male and fifteen female, 9 to 10 week old, axenic eaesarian-derived (CD) rats (Charles River Breeding Laboratories, Wilmington, Mass.), 150-180 $\mathrm{g}$ in weight, were housed in sterile isolators and were fed sterile rat food and water ad libitum. Isolators were monitored for bacterial and fungal contamination by repeated sampling of feces, bedding and water bottles. They remained sterile throughout the experiment.

Seed PRCV was obtained from Dr. John C. Parker, Mierobiological Associates, Bethesda, Maryland. Virus stock was prepared by one passage in primary rat kidney (PRK) cultures prepared from a germfree CD rat as previously deseribed (2). Aliquots of infected tissue culture fluid were stored at $-60^{\circ} \mathrm{C}$ with equal volumes of fetal bovine serum (FBS). Unanesthetized rats were inoculated intranasally with $0.1 \mathrm{ml}$ of culture fluid containing $4.6 \log _{10}$ TCID $_{50}$ of PRCV. Culture fluid was removed from isolators immediately after inoculation and was retitered for infectious PRCV in PRK monolayers as previously described (2).

On each of eight consecutive days after inoculation three to four rats, selected at random, were anesthetized with pentobarbital sodium and exsanguinated. Serum was stored at $-60^{\circ} \mathrm{C}$. Sections of trachea, lung, cervical lymph node, submaxillary and parotid salivary glands and exorbital and Harderian lacrimal glands were harvested aseptically and were frozen at $-60^{\circ} \mathrm{C}$. Nasopharyngeal cavities were irrigated with sterile minimum essential medium in Hank's base containing 50 per cent FBS (MEMHFBS) and washings were also frozen at $-60^{\circ} \mathrm{C}$. Tissues were homogenized (10 per cent $\mathrm{w} / \mathrm{v}$ ) in MEMH-FBS and were clarified by lowspeed centrifugation. Supernatants were serially diluted and inoculated into PRK oultures. Observations for cytopathic effects were carried out as previously reported (2) and endpoints were calculated as described by REED and MUENCH (8). Virus in lungs, tracheas, nasal washings, salivary glands and lacrimal glands was quantitated for individual rats. Lymph nodes were pooled by collection day before being titrated. Anti-PRCV immune serum and PRCV CF antigen were donated by Drs. John Parker and Michael Collins of Microbiological Associates, Bethesda. Serum neutralizing (SN) and CF antibody titers to SDAV and PRCV were determined as previously described (2).

Sections of nasal turbinate, trachea, cervical lymph node, submaxillary and parotid salivary gland and exorbital and Harderian lacrimal glands were snap frozen in dry icealeohol baths. Lungs were inflated with 7.5 per cent gelatin and placed at $0^{\circ} \mathrm{C}$ for one hour to gel, sliced and frozen on chueks at $-20^{\circ} \mathrm{C}$. Rat anti-SDAV IgG was prepared and aliquots were conjugated to fluorescein isothiocyanate (3). Preliminary tests indicated that anti-SDAV and anti-PRCV sera gave equally good results for indirect immunofluorescent staining of PRCV-infected tissues. Goat anti-mouse globulinfluorescein isothiocyanate (Lot AA 720073, Antibodies, Incorporated, Davis, California) was used with mouse anti-SDAV immune ascitic-fluid for indirect staining. Sections of snap frozen tissues $6 \mathrm{~mm}$ thick were fixed overnight in acetone at $-20^{\circ} \mathrm{C}$ and were stained by the direct and/or indirect fluorescent antibody technic. They were examined with a Zeiss microseope fitted with an HBO 200 Osram lamp, a BG-12 ultraviolet exciter filter and numbers 44 and 53 barrier filters.

Coronal sections of nasopharynx at three levels and sections of trachea, lung (inflated with fixative via the trachea), cervical lymph node, salivary glands, lacrimal glands, heart, thymus, liver, spleen, kidney, panereas, adrenal gland, gonad, eye and brain were fixed in 10 per cent neutral buffered formalin, sectioned at $6 \mu \mathrm{m}$, stained with hematoxylin-eosin and examined by light microscopy. 


\section{Results}

Rats were free of detectable clinical disease during the eight day experiment. PRCV was recovered from nasal washes and trachea by day one, from cervical lymph nodes by day two and from lung by day three (Fig. 1). Titers were usually higher in trachea than in lung. Virus was rarely detected in salivary or lacrimal glands and was not found in serum. Virus was not detected in nasopharynx, trachea and lung after day six and in other tissues after day seven except in the submaxillary salivary gland of one rat.

Intracytoplasmic viral antigen was detected by immunofluorescence in epithelial cells of nasal mucosa by day two. Fluorescence was multifocal and persisted until day six when only several small foci of antigen remained. Tracheal fluores-

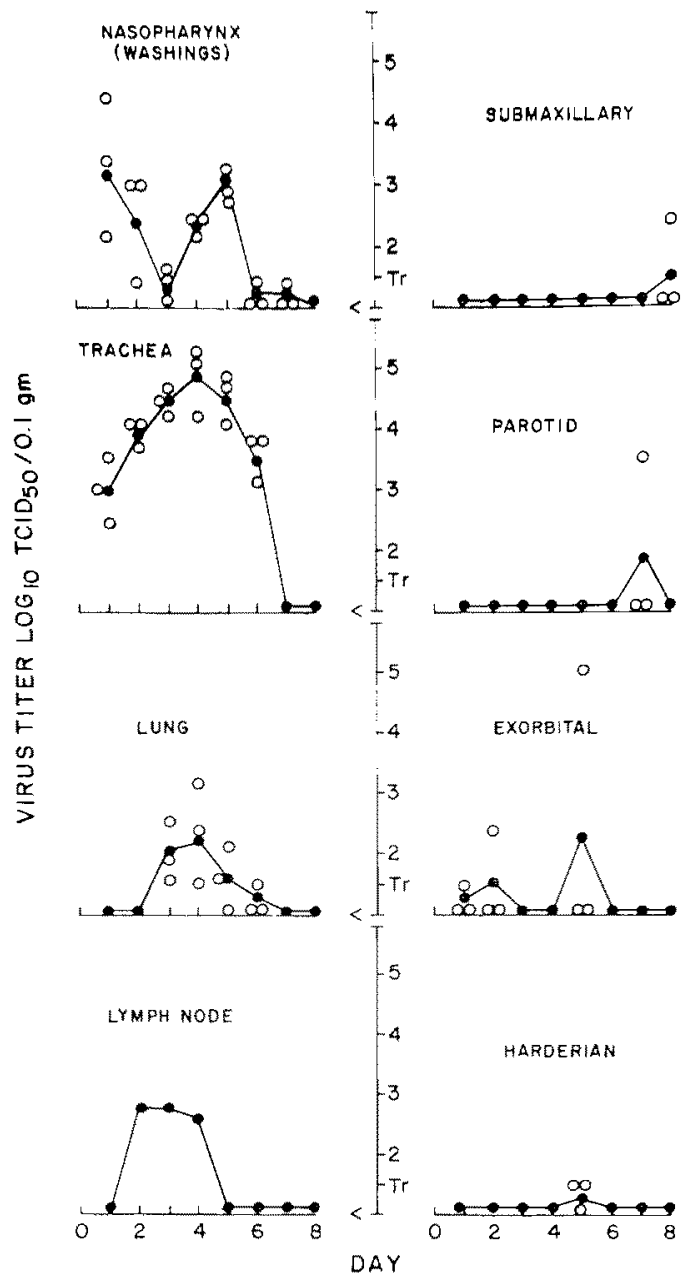

Fig. 1. Thirty axenic rats nine to ten weeks old, were inoculated intranasally with 104.6 TCID 50 of PRCV. Three to four rats were necropsied each day for eight days. Virus was quantitated in tissues shown. Open circles are individual titers. Closed circles indicate average titers. Lymph nodes were pooled 
cence was sparse; a few mucosal epithelial cells contained viral antigen on days two through four. Fluorescence in lung was also sparse and was observed only on days six and seven. Antigen was not detected in parotid, submaxillary, exorbital and Harderian glands or in cervical lymph nodes.

Gross lesions were confined to lung, were seen on days six and seven and consisted of several small (less than $1 \mathrm{~mm}$ ) red-brown to gray foci which were randomly dispersed over all lobes. A few lungs had patchy areas of pale red to gray discoloration on individual lobes, but the lobes were not firm.

Histologically, lesions occurred primarily in the respiratory system and were first seen on day two as mild rhinitis. There was multifocal or segmental necrosis of respiratory epithelium covering nasal turbinates. The lamina propria was mildly edematous and contained lymphocytes and a few neutrophils which occasionally infiltrated interstitial tissues of underlying glands. Some meatuses contained neutrophils and cell debris. Similar lesions were found through day four (Figs. 2, 3) although there was a relative increase in the proportion of neutrophils in the lamina propria and several necrotic acini were seen among submucosal glands. Hyperplasia of paraseptal lymphoid tissue was noticed by day four, but germinal centers were not present.

By day five acute rhinitis was accompanied by mild nonsuppurative tracheitis. Tracheal lamina propria had infiltrates of lymphoid cells and some neutrophils. Neutrophils were also found in mucosal epithelium but epithelial necrosis was generally sparse. Occasionally inflammation was more severe and included substantial necrosis of epithelium and tracheal glands.

Lung lesions began by day five as focal peribronchial lymphoid cell hyperplasia and mild focal interstitial pneumonia. Alveolar septae contained mononuclear cells and neutrophils and there were some inflammatory cells in adjacent

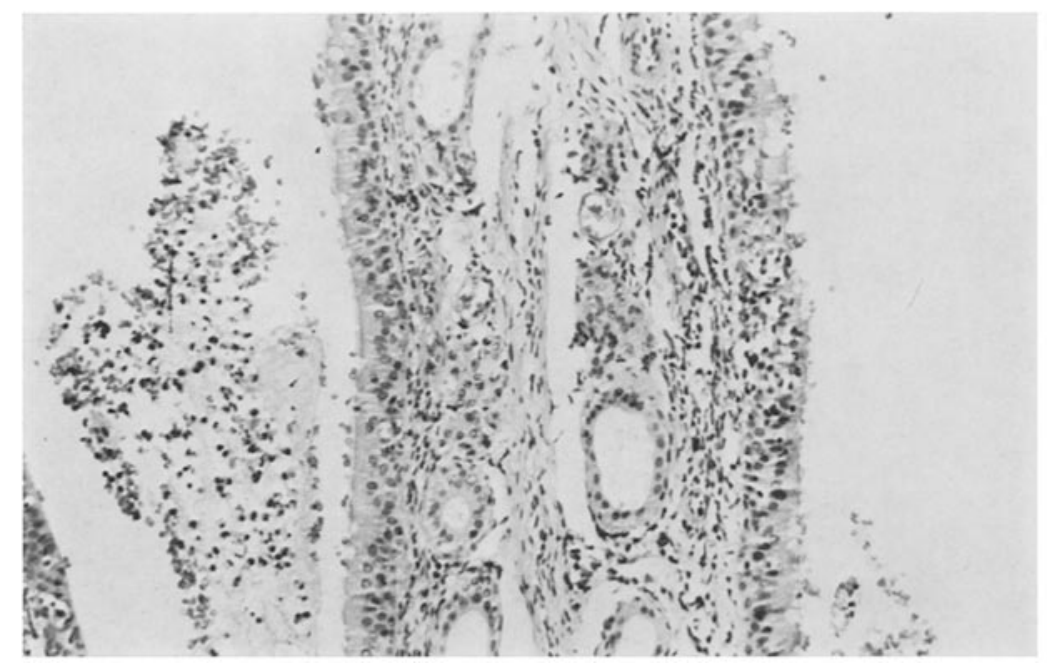

Fig. 2. Nasal cavity 4 days after intranasal inoculation with PRCV. There is necrosis of mucosal epithelium, inflammatory edema of the lamina propria and exudation of mucus and neutrophils into adjacent meatuses. The erythrocytes accumulated in meatuses during the gross dissection 
alveolar spaces (Figs. 4, 5). By day six pneumocytes, foamy macrophages and edema fluid partially filled some alveoli, but lesions remained mild and focal. There were also traces of nonsuppurative perivasculitis in several lungs. Necrosis of bronchial epithelium was not observed. Nasopharyngeal and tracheal inflammation subsided by day seven and only traces of rhinitis remained by day eight. Pulmonary lesions were not detected on day eight.

Salivary gland lesions were rare. Parotitis was found in two rats on day seven and submaxillary sialoadenitis was found in one rat on day eight. Lesions were

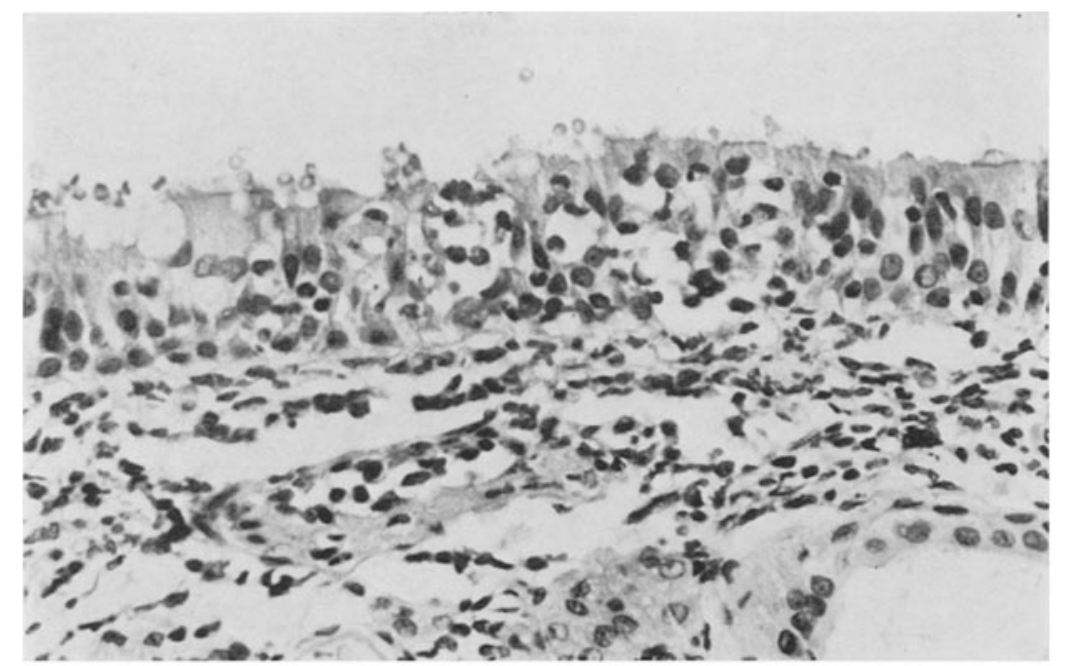

Fig. 3. Higher magnification of nasal mucosa from figure showing epithelial necrosis with infiltration of epithelial layer and lamina propria by lymphoid cells and neutrophils

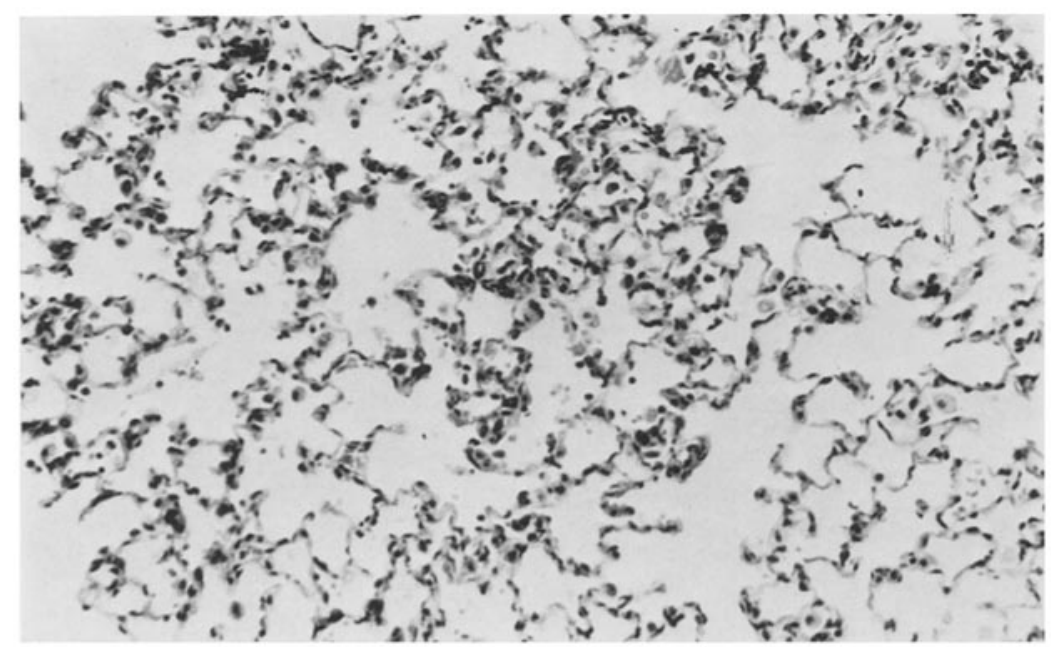

Fig. 4. Lung 5 days after intranasal inoculation with PRCV. There is interstitial pneumonia characterized by infiltration of alveolar septae with mononuclear cells. Alveolar spaces contain a few macrophages 
identical to those caused by $\operatorname{SDAV}(4,5)$ and were characterized by necrosis of salivary ducts with periductular and interstitial inflammatory edema. There were no lesions in lacrimal glands, eye, liver, spleen, heart, kidney, thymus, cervical lymph node, adrenal gland, gonad, or brain.

Anti-PRCV and anti-SDAV neutralizing antibody were detected by day six and seven, respectively (Table 1). CF antibody to PRCV and SDAV was not detected through day eight.

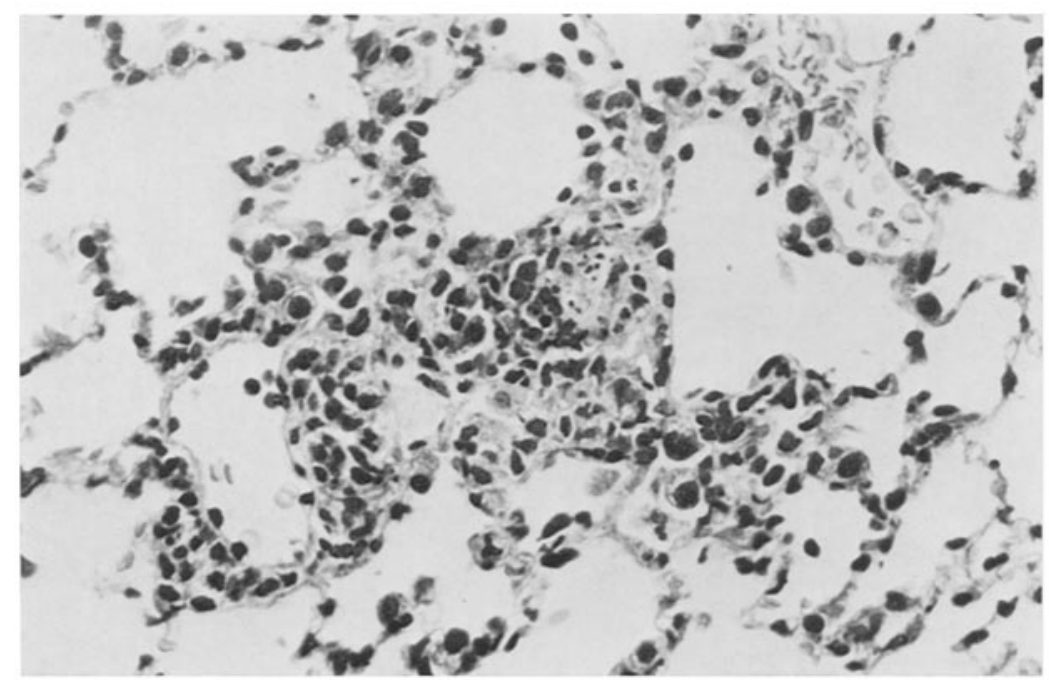

Fig. 5. Lung from a rat with interstitial pneumonia 6 days after intranasal inoculation with PRCV. Vein at left is surrounded by inflammatory cells and alveolar septae are also infiltrated. There is a small area of necrosis in the center of the field

Table 1. Serum neutralizing antibody titers to PROV and SDAV in germfree rats experimentally inoculated with $P R C V$

\begin{tabular}{|c|c|c|c|c|c|c|c|c|c|}
\hline \multirow[b]{2}{*}{ Virus } & \multirow[b]{2}{*}{ Antibody titer } & \multicolumn{8}{|c|}{ Postinoculation day } \\
\hline & & 1 & 2 & 3 & 4 & 5 & 6 & 7 & 8 \\
\hline \multirow{4}{*}{ PRCV } & $\begin{array}{r}<10 \\
10\end{array}$ & $3^{\mathrm{a}}$ & 4 & 4 & 4 & 4 & & & \\
\hline & 20 & & & & & & 2 & & \\
\hline & 40 & & & & & & & & \\
\hline & $\geqslant 80$ & & & & & & 2 & 4 & 3 \\
\hline \multirow{4}{*}{ SDAV } & $\begin{array}{r}<10 \\
10\end{array}$ & $\mathrm{ND}^{b}$ & ND & $\mathrm{ND}$ & $\mathrm{ND}$ & $\mathrm{ND}$ & 4 & & \\
\hline & 20 & & & & & & & 3 & \\
\hline & 40 & & & & & & & 1 & 1 \\
\hline & $\geq 80$ & & & & & & & & 2 \\
\hline
\end{tabular}

a Number of individual sera tested

b $\mathrm{ND}=$ Not Done 


\section{Diseussion}

Parker and coworkers previously demonstrated that PRCV caused lethal interstitial pneumonia in experimentally infected suckling rats and they suggested that PRCV could play a role in chronic respiratory disease of adult rats (7). We extended their hypothesis to include SDAV by showing it was also a primary pathogen for the respiratory system of adult rats (4). Experiments reported here show directly that PRCV infection of adult rats, although asymptomatic, causes enough inflammation of the respiratory tract to warrant continued scrutiny as an initiator or copathogen in clinically severe respiratory syndromes.

It is clear that PRCV and SDAV are closely related viruses. Their antigenic similarity has been well documented, but in cross neutralization tests titers to the homologous virus were persistently higher than to the heterologous virus (2). Similar results were obtained in the present experiment where PRCV-infected rats had SN antibody to PRCV by day six and slightly lower SN titers to SDAV by day seven.

It is of interest in this regard that PRCV and SDAV were originally isolated from lungs of Fisher rats and salivary glands of CD Sprague Dawley rats, respectively. These rats were raised in different rooms of the same animal facility.

Strains of mouse hepatitis virus (MHV) (Coronavirus) differ in their pathogenicity and tissue tropism although they are closely related serologically (1). Our findings indicate that the relationship between SDAV and PRCV is similar to that among MHV strains. Therefore, we propose that SDAV and PRCV be considered different strains of rat coronavirus.

When the pathobiology of SDAV (4) and PRCV infection in vivo is compared, additional differences emerge (Table 2). First, clinical signs of rhinitis and sialo-

Table 2. Comparison of the major features of experimental infection with PRCV and $S D A V$ in adult axenic $C D$ rats

\begin{tabular}{lll}
\hline Feature & SDAV & PRCV \\
\hline Clinical signs & & \\
Photophobia & Yes & No \\
Sneezing & Yes & No \\
Cervical swelling & Yes & No \\
Virus Replication & & \\
Respiratory system & Yes & Yes \\
Salivary glands & Yes & Trace \\
Lacrimal glands & Yes & Trace \\
Losions & & \\
Acute rhinotracheitis & Yes & Yes \\
Focal interstitial pneumonia & No & Yes \\
Sialoadenitis & Yes & Trace \\
Dacryoadenitis & Yes & No \\
Antibody response & & \\
Complement fixing & Yes & No \\
Neutralizing & Yes & Yes \\
\hline
\end{tabular}

a Up to eight days post inoculation CF antibody can develop if rats are tested at later times 
dacryoadenitis were observed frequently during experimental SDAV infection whereas PRCV infection in rats of the same strain and of the same age and source was asymptomatic. Second, the tissue tropism of PRCV differed from SDAV. Both viruses replicated in the respiratory tract and caused rhinotracheitis but PRCV also caused mild pneumonia whereas SDAV did not. Conversely, PRCV replicated poorly in salivary and lacrimal glands and only rarely produced lesions, whereas SDAV was severely pathogenic for these glands. Nevertheless, sialoadenitis produced by PRCV, although mild when it occurred, was morphologically compatible with SDAV-induced lesions. The potential for PRCV-induced sialoadenitis may be mitigated by factors such as strain, age and sex of the host. Additional experiments should examine the effects these variables have on coronavirus infections of rats.

This study also underscores the usefulness of documenting and correlating virological and morphological data to assess the pathogenetic significance of infections which are clinically silent and which are usually detected only by serological monitoring.

\section{Acknowledgements}

Supported by PHS Grants RR00700, RR00393 and FR05358.

The authors wish to acknowledge excellent technical assistance of Miss M. Nettleton, Miss B. Collett and Miss D. Davis.

\section{References}

1. Andrewes, C., Pereira, H. G.: Mouse Hepatitis Virus (MHV), Viruses of Vertebrates, 181-182. Baltimore: Williams \& Wilkins 1972.

2. Bhatt, P. N., Percy, D. H., Jonas, A. M.: Characterization of the Virus of Sialodacryoadenitis of Rats: A Member of the Coronavirus Group. J.inf. Dis. 126, 123130 (1972).

3. Cebra, J. J., Goldstein, G.: Chromatographic purification of tetramethylrhodamine immune globulin conjugates and their use in the cellular localization of rabbit gammaglobulin peptide chains. J. Immunol, 95, 230-245 (1965).

4. JACOBY, R. O., Bhatt, P. N., Jonas, A. M. : Pathogenesis of Sialodacryoadenitis in Gnotobiotic Rats. Vet. Pathol. 12, 196-209 (1975).

5. Jonas, A. M., Craft, J., Black, D. L., Bhatt, P. N., Hilding, D.: Sialodacryoadenitis in the Rat (A Light and Electron Miscrocopic Study). Arch. Pathol. 88, $613-622(1969)$.

6. LAI, Y. L., JACOBY, R. O., BHATT, P. N., Jonas, A. M.: Keratoconjunetivitis associated with sialodacryoadenitis in rats. Invest. Ophth. 15, 538-541 (1976).

7. Parkrer, J. C., Cross, S. S., Rowe, W. P.: Rat Coronavirus (RCV): A Prevalent, Naturally Occurring Pneumotropic Virus of Rats. Arch. ges. Virusforsch. 31, 293$302(1970)$.

8. ReED, L. J., MUench, H. A.: A simple method of estimating fifty percent and endpoints. Amer. J. Hyg. 27, 493-497 (1938).

Authors' address: Dr. P. N. BHATt, Laboratory of Virology and Epidemiology, Section of Comparative Medicine, Yale University School of Medicine, 375 Congress Avenue, New Haven, CT 06510, U.S.A. 